\title{
A sensitive test for tactile extinction: results in patients with parietal and frontal lobe disease
}

\author{
A. S. SCHWARTZ ${ }^{1}$, PATRICIA L. MARCHOK, AND R. E. FLYNN \\ From the Divisions of Neurobiology and Neuroradiology, Barrow Neurological Institute of St. Joseph's \\ Hospital, and Medical Center, Phoenix, Arizona 85013, USA
}

SUMMARY A simple test for detecting tactile extinction is described. In a population of parietaldamaged patients it yielded fewer false negatives than the classical clinical procedure. Contrary to expectations, lesions confined to the right frontal lobe produced no extinction, while those in right- in handed, left frontal cases revealed ipsilateral extinction with the new test.

The phenomenon of tactile extinction-that is, the failure to detect a stimulus only in the presence of another stimulus to certain parts of the body-is a well-known clinical sign of parietal lobe disease (Nathan, 1946; Critchley, 1949, 1953; Bender, 1952). More recently Heilman and Valenstein (1972) have reported that extinction may also occur in patients with isolated frontal lobe damage. Monkeys demonstrate tactile extinction after frontal as well as parietal lesions (Schwartz and Eidelberg, 1968; Eidelberg and Schwartz, 1971), and unilateral neglect has been observed in the monkey (Welch and Stuteville, 1958) and the human (Heilman and Valenstein, 1972) with focal damage to the frontal lobe.

In our experience, the classical tactile extinction testing procedure (light touch with the fingers to the subject's hands or face) has proved unreliable in terms of the proportion of false negatives among patients with documented parietal or frontal lobe damage. In at least one published report on patients with unilateral focal lesions (unspecified), only $12 \%$ made more than two errors on double simultaneous stimulation (Gainotti et al., 1975). The consideration that the classical procedure may be too crude for revealing tactile extinction prompted us to attempt to construct a more sensitive tactile discrimination test for extinction and to compare the occurrence of this phenomenon in patients with parietal as opposed to extraparietal lesions. We describe here such a test, together with preliminary data on a group of relatively unselected cases with supratentorial disease.

1 Address for correspondence: Division of Neurobiology. Barrow Neurological Institute, 350 West Thomas Road, Phoenix, Arizona 85013, USA.

(Accepted 11 October 1976.)

\section{Method}

Forty normal control subjects and 76 neurological is patients were examined for extinction. The normati controls were mostly hospital employees and offere $\Phi$ i no history of past or present neurological symptoms $\infty$ Their ages ranged from 20 to 65 years (mean $=3 \overline{6}$ 음 years); 18 were males and 22 were females.

The patient population was divided into two groups. The first comprised an additional contro group and consisted of those patients with no curren evidence of impairment of central nervous structure $\vec{\theta}$ rostral to $L 1$ segment of the spinal cord. The second $\overrightarrow{ }$ (or 'experimental') group consisted of patients with supratentorial pathology confirmed by radiological and/or surgical means. The experimental subjects were relatively unselected except that we excluded those with aphasia, short-term memory loss, unilateral hypaesthesia or neglect-that is, unable to identify single tactile stimulation to one side of the body, see Discussion-or bilateral cerebral pathology. There were 10 males and 16 females, ranging in age from 20 to 70 years (mean $=47$ years) among the control patients, and 28 males and 22 females ranging from 13 to 76 years (mean $=49$ years) among the experimental patients.

The criteria for diagnostic classifications were derived from histories, clinical examinations, angiographies, radionuclide or computerised axial tomography (CT) scans, and visualisation during surgery. The battery of tests of sensation was administered without knowledge of the compromised hemisphere in most cases. The sensory tests, in order of presentation, consisted of the classical tactile extinction test with the experimenter's fingers, and our experimental Quality Extinction Test (QET). 
For the QET we reasoned that extinction during bilateral simultaneous stimulation would be enhanced if a more complex discrimination were required than the presence or absence of a simple touch as in the classical technique. Accordingly, an assortment of materials differing in tactile qualities was assembled such as carpeting, sandpaper, velvet cloth, wire screening, foam rubber, and a paint-roller. These items were mounted individually on a stiff, semicircular backing $20 \mathrm{~cm}$ long and $8 \mathrm{~cm}$ outer circumference. Another set was constructed consisting of these same materials, but mounted so that half the length of each test object was of one material and a different material covered the other half, in various combinations. Thus one test object had sandpaper for half its length and wire screen for the other, while another consisted of half sandpaper and half velvet, etc. The former items are termed the 'complete' set, while the latter constitute the 'half-and-half' set.

The patient was told that we wished to examine him with an experimental battery of sensory tests. He was shown only the 'complete' QET items and asked to handle and identify them so that he could recognise and name them later while blindfolded. Once the subject could identify each material by a combination of both visual and tactile observation-that is, no memory or speech deficit being present-the blindfold was applied and testing began.

The classical extinction examination was presented first, and involved 20 trials of single or double simultaneous light touches to the face or hands, using the experimenter's fingers. The subject was next told that he would be tested with the objects shown earlier, the implication being that the objects would consist entirely of the complete set which he had examined. The first objective was to establish that each hand alone had the requisite sensitivity for discrimination of the various materials. He was instructed to hold out his left hand, palmar surface up and fingers extended; each complete object was then brushed lightly against the fingers in a proximal-distal direction at a speed of about $5 \mathrm{~cm} / \mathrm{s}$, followed by testing of the right hand. If an item could not be identified after several trials, or if one hand was hypaesthetic relative to the other, the data were discarded.

After testing for unilateral sensitivity, both hands were placed side by side, palms up as before and about $4 \mathrm{~cm}$ apart. Each complete object was now applied to both hands simultaneously for six trials. At this point the half-and-half objects were introduced; they were brushed against both hands in the same manner as the complete items except that each hand was stimulated by a different material simultaneously. This latter procedure constituted the Quality Extinction Test, and consisted of a mixed sequence of both half- - damaged population is shown in Tables 1 and 2 , the and-half and complete items for 24 trials. If the sub-first table including those manifesting parietal lobe ject questioned whether there had been two qualities, he was asked to name and locate them. Each naming response, whether correct or incorrect, was reinforced by the examiner with a 'Good' or other term of approval. Never was the subject told that, indeed, there were two separate qualities. By definition, an extinction response was scored when the subject responded to a half-and-half item by naming only one of the qualities. Thus, if a trial consisted of the wire-foam object stimulating the left and right hand respectively, and the subject responded 'Foam', the trial was scored as a left extinction response. QET scores were computed for each subject by subtracting the number of extinction responses (in terms of per cent of total half-and-half stimulation trials) on one side from the per cent extinction responses on the other. For example, a subject with $70 \%$ extinction responses of the left side and $20 \%$ of the right would receive a QET score of $50 \%$, left side, while $50 \%$ right extinction responses and $50 \%$ left extinction responses would yield a QET score of $0 \%$.

\section{Results}

Normal control subjects usually detected the halfand-half qualities immediately or soon after a few presentations. They either reported correctly or extinguished on both sides with comparable frequency so that their median QET score was $4.0 \%$. Fifteen extinguished more on the left and 13 more on the right; there was no relationship to handedness. The highest score was $26 \%$, obtained from a 23 year old male who was re-examined one week later; his score then was $4 \%$. We have no explanation for the high score in a presumably normal individual, other than that the QET may be a relatively severe test which can yield a low incidence of extinction among normal subjects. The next highest score was $14 \%$, followed by two scores of $11 \%$ (see second column, Table 1).

The patient control group showed a similar QET distribution (third column, Table 1), although the median score was higher than the normal group $(9.0 \%)$. The difference between the two control groups was not significant $\left(\chi^{2}=2.437\right.$; df $=1$; $\mathrm{P}>0.10$ ). One patient, a 46 year old female who complained of blackouts but showed no evidence of cerebral pathology on radiographic or clinical examination, yielded a QET score of $31 \%$, left. She was diagnosed as a possible hysteric; she could not be followed up. None of the other patients in this group scored higher than 20 , and their test performance resembled quite closely that of the normal group.

The QET score distribution among the brain- 
Table 1 Frequency distribution of QET scores and positive classical extinction in control groups and parietal lobe patients

\begin{tabular}{|c|c|c|c|c|}
\hline \multirow[b]{2}{*}{$\begin{array}{l}Q E T \\
\text { scores } \\
(\%)\end{array}$} & \multicolumn{2}{|c|}{ Number of control subjects } & \multicolumn{2}{|c|}{$\begin{array}{l}\text { Parietal patients } \\
(N=34)\end{array}$} \\
\hline & $\begin{array}{l}\text { Normal } \\
\text { subjects } \\
(N=40)\end{array}$ & $\begin{array}{l}\text { Neurological } \\
\text { patients } \\
(N=26)\end{array}$ & $Q E T$ & $\begin{array}{l}\text { Classical } \\
\text { extinction }\end{array}$ \\
\hline $0-10$ & 36 & 16 & 1 & 0 \\
\hline $11-20$ & 3 & 9 & 1 & 0 \\
\hline $21-30$ & 1 & 0 & 4 & 0 \\
\hline $31-40$ & 0 & 1 & 1 & 0 \\
\hline $41-50$ & 0 & 0 & 2 & 1 \\
\hline $51-60$ & 0 & 0 & 5 & 2 \\
\hline $61-70$ & 0 & 0 & $6^{*}$ & 4 \\
\hline $71-80$ & 0 & 0 & 5 & 2 \\
\hline $81-90$ & 0 & 0 & 6 & 5 \\
\hline $91-100$ & 0 & 0 & 3 & 1 \\
\hline
\end{tabular}

*Includes one patient with ipsilateral QET extinction (see text). Al other cases showed extinction on side contralateral to lesion.

Table 2 QET and classical extinction results in non-parietal patients

\begin{tabular}{|c|c|c|c|}
\hline $\begin{array}{l}\text { Case } \\
\text { no. }\end{array}$ & Diagnosis & $\begin{array}{l}Q E T \\
\text { score } \\
(\%)\end{array}$ & $\begin{array}{l}\text { Classical } \\
\text { extinction }\end{array}$ \\
\hline 1 & L Frontal meningioma & L 70 & - \\
\hline 2 & R Frontal astrocytoma & L $\quad 5$ & 一 \\
\hline 3 & $\begin{array}{c}\text { R Frontal, anterior } \\
\text { glioblastoma }\end{array}$ & L $\quad 17$ & - \\
\hline 4 & R Frontobasal tumour & L $\quad 14$ & 一 \\
\hline 5 & L Frontal, probable infarct & L 62 & - \\
\hline 6 & $\begin{array}{l}\text { L Frontal astrocytoma, } \\
1 \mathrm{~m} \text { post-operative }\end{array}$ & 0 & - \\
\hline 7 & L Frontal porencephalic cyst & L 36 & - \\
\hline 21 & $\begin{array}{l}\text { L Temporal astrocytoma, } \\
\text { recurrent }\end{array}$ & 0 & - \\
\hline 22 & $\mathbf{R}$ Temporal astrocytoma & L 7 & - \\
\hline 23 & L Temporal astrocytoma & R 11 & - \\
\hline 24 & L Temporal glioblastoma & R 23 & - \\
\hline 25 & $\begin{array}{l}\text { R Temporal-occipital } \\
\text { glioblastoma }\end{array}$ & L 20 & - \\
\hline 41 & $\begin{array}{l}\text { R Occipital lobectomy, } \\
\text { metastatic tumour }\end{array}$ & 0 & - \\
\hline 42 & $\begin{array}{l}\text { R Occipital lobectomy } \\
\text { glioblastoma }\end{array}$ & 0 & - \\
\hline 43 & L Occipital infarct & $\mathbf{L}$ & - \\
\hline 44 & $\mathbf{R}$ Occipital infarct & $\mathbf{L}$ & - \\
\hline
\end{tabular}

-: negative classical extinction.

L: left side.

R: right side.

lesions and the second table those without any demonstrable parietal disease. Clearly the test's greatest sensitivity is in discriminating patients with parietal lobe disease from normal subjects and from cases with non-cerebral involvement. Only one of the parietal patients scored lower than $20 \%$ (fourth column, Table 1), while all but two of the controls did so. The median scores of the controls and the parietal patient groups were $5 \%$ and $58 \%$ respectively; the difference was statistically significant $(\mathrm{P}<0.001)$. Fourteen parietal patients extinguished the right side (with left hemisphere lesions) and 19 extinguished the left side (right hemisphere lesions). However, one patient with a metastatic tumour extending from the frontal to the occipital lobe of the left hemisphere obtained a left QET score of $66 \%$, without extinguishing once by the classical extinction test. This and three other cases of ipsilateral QET extinction will be discussed further below. CT scans of the single patient classified as having a right parietal lobe lesion but scoring well within the normal range on both the classical extinction test and the QET (L 5\%) are shown in Fig. 1.

The classical extinction examination proved less sensitive than the QET (fifth column, Table 1). None of the control subjects made more than one error on bilateral homologous stimulation; two such errors was therefore considered positive. Based on this criterion, 19 of the parietal patients were negative while 15 were positive. During the classical examination we also tested for face dominance and displacement, according to the method of Bender (1952). Of the 19 parietal lobe patients who were negative in terms of bilateral, simultaneous stimulation of homologous areas, four of these made more displacement and/or face-dominance errors than the control popu- $\frac{}{8}$ lation. Thus $44 \%$ of the parietal group scored within the normal range on the several aspects of the classicalo extinction examination. The CT scan of one such patient is shown in Fig. 2; this 13 year old male maden no errors during the classical extinction test, but one the QET he extinguished the left quality 12 times, the right quality once, and responded correctly to both qualities on six trials, for a QET score of $55 \%$. At the time of surgery an $8 \times 8 \times 2 \mathrm{~cm}$ ependymoma within the right parieto-occipital region and extending through the atrium of the right lateral ventricle was removed.

Several non-parietal patients who met our earlierstated criteria were also examined with the extinction test battery (Table 2). Two items of interest emerge from these data. First, none of these patients extinguished with the classical procedure. Second, none of the frontal patients extinguished on the side contralateral to their lesion, but, most unexpectedly, three patients (cases 1,5 , and 7, Table 2) with lesions confined to the left frontal lobe showed a clearly positive QET score of the ipsilateral side. This anomalous result was also obtained in a parietal patient whose CT scan revealed metastatic tumour extending from the frontal to the occipital lobes on the left (see footnote, Table 1). Repeat testing of another case (no. 5) produced a QET result of $100 \%$, again ipsilateral. All four had damage confined to the left hemisphere, and all four were right handed; three were females. None of these anomalous patients was grossly aphasic. A possible exception to ipsilateral 


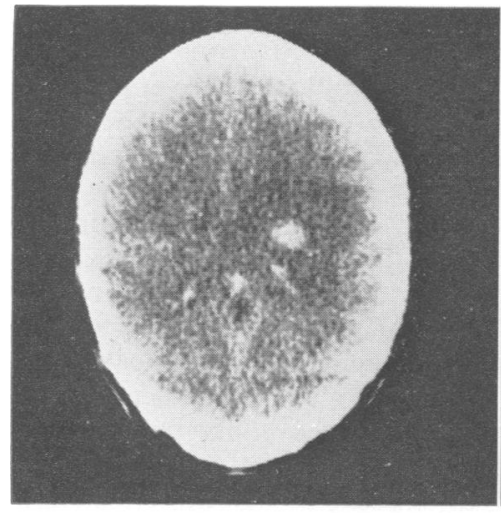

(a)

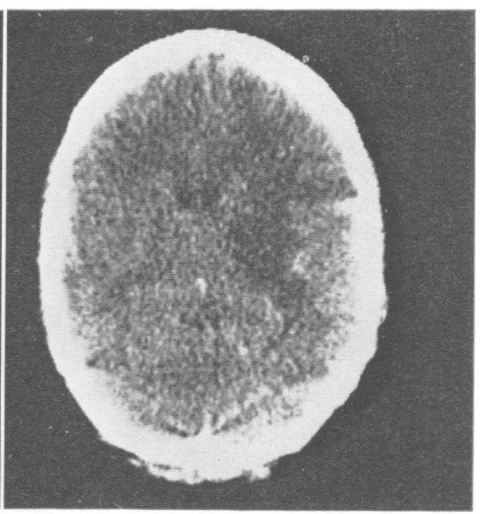

(b)

Fig. $1 C T$ scans of the only patient in this series classified as a parietal case but who showed no extinction with either the classical procedure or the QET. (a) High density mass at thalamic level, below parietal lobe. (b) Low density lesion, probably oedema, extending upward into right posterior fronto-parietal region. (By courtesy of Dr Robert S. Waldman, Computed Tomography Ltd., Phoenix.)

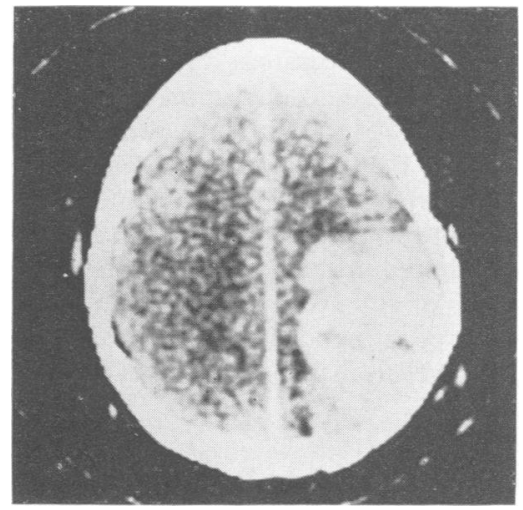

Fig. 2 CT scan of 13 year old male with right recurrent ependymoma. Patient performed normally on classical extinction while his QET was positive.

QET extinction in a left frontal, right hander, may be represented by case 6 , Table 2 . This patient scored normally when tested as an outpatient one month after surgery, whereas the ipsilateral extinguishers were tested within eight days or before surgery. The possibility that case 6 may also have extinguished ipsilaterally before removal of her tumour cannot be excluded (Krueger, et al., 1954; see Teuber, 1975). CT scans of a case with ipsilateral QET extinction and of a non-extinguishing right frontal patient are shown in Fig. 3 and Fig. 4 respectively.

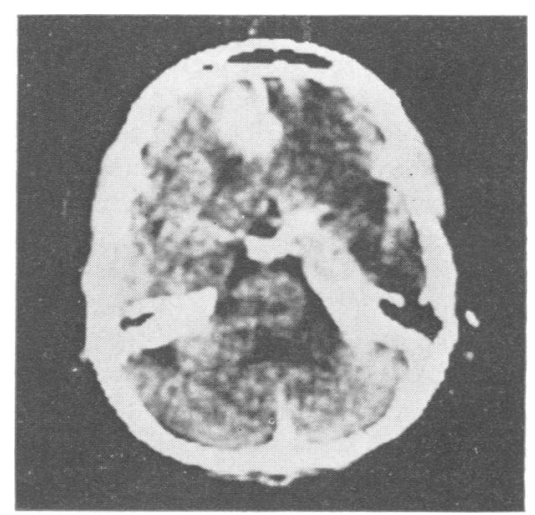

Fig. 3 CT scan showing mass in left frontal lobe of right-handed female. Negative classical extinction, positive QET on left side.

\section{Discussion}

One aspect of the extinction phenomenon is the lack of attention to or perception of a stimulus when a contralateral stimulus is simultaneously presented, even though the unreported stimulus is perceived adequately when presented alone (Bender, 1952; Critchley, 1953). The QET described here conforms with this definition and is demonstrably more sensitive to unilateral parietal lobe damage than the classical extinction test procedure. Although more 


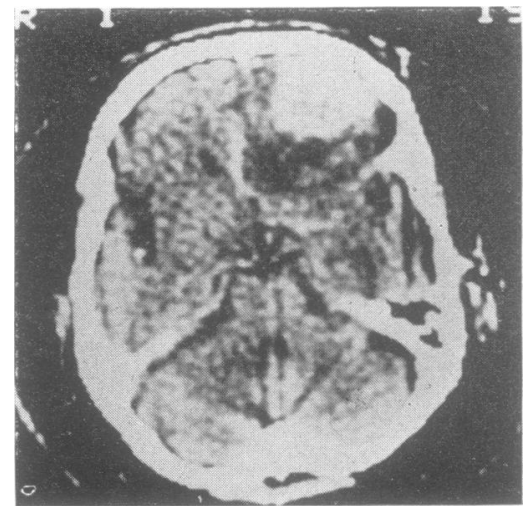

Fig. 4 Case 3. CT scan showing tumour in right frontal lobe of right-handed male. Classical extinction and QET both negative.

experience with the QET and anatomical verification is needed before establishing a cut-off score with confidence, the present results indicate that a QET score above $20 \%$ would be grounds for suspecting a deficit in the contralateral parietal lobe. Only one patient, or $3 \%$ of the total number of parietal lobe cases in our sample, scored below this point, while the classical extinction procedure yielded a false-negative incidence of $44 \%$ when including face-dominance errors, and $56 \%$ (see Table 1) when considering only errors during bilateral homologous stimulation.

In the light of the demonstrated sensitivity of the QET in parietal lobe cases, it is surprising that only three out of the seven patients with focal frontal lesions achieved a positive score (Table 2), and even more surprising that these three (plus one case with combined frontal and parietal involvement) manifested paradoxical extinction of the side ipsilateral to their lesion. Earlier work on monkeys found tactile extinction after subtotal frontal ablations (Schwartz and Eidelberg, 1968; Eidelberg and Schwartz, 1971), and has been reported in the human with presumably isolated frontal lobe disease (Heilman and Valenstein, 1972). The latter study does not indicate the number of frontal cases showing no (classical) extinction, or whether the patients' unilateral neglect served to contaminate the extinction test. In our experience, unilateral tactile neglect is rare and was not observed even among the present patients with extensive parietal and frontal lesions. Although extinction (which apparently is an active process, since by definition it is triggered only by the presentation of a second, simultaneous stimulus) and unilateral neglect may share certain mechanisms, they have not been proven to be identical, or even that neglect represents an extreme condition of extinction. Despite the extensive literature on unilateral (tactile) neglect we are not aware of a single case of extinction of bilateral simultaneous tactile stimulation in a human with pathologically verified isolated frontal lobe damage. Our own frontal cases are no exception, as postmortem verification that the lesions were limited to the frontal lobe at time of testing was not obtained. All these considerations suggest the possibility that the human differs from the monkey in that contralateral tactile extinction is a specific characteristic of damage to the parietal lobe and its input components in the former species only, whereas extinction may be evident with more widespread damage in the monkey.

The unexpected findings of ipsilateral extinction in all three patients with relatively acute left frontal lesions, and one with combined left frontal and parietal damage, indicates that a positive left QET score need not reflect right parietal disease. We will not speculate about the possible mechanism for ipsilateral QET extinction until further data are obtained, but it would seem to be related to the function of the dominant (speech) hemisphere, and this may underlie the species differences noted above.

A final note may be mentioned regarding the $\omega$ utility of sensitive neuropsychological tests such aO N the QET as a refinement of clinical examination pro cedures. On several occasions a positive QET finding in a case initially diagnosed as non-parietal has led tQ a re-examination of the patient's radiographic datao with the result that a space-occupying lesion in the parietal area was discovered or considered likely This observation emphasises again the fallacy of relying on limited criteria in determining the neurological status of any given case. The simplicity of the QET may recommend its usefulness in clinical diagnosis, as well as in identification of the mechansim of extinction.

This project was supported in part by GRS Grant RR 05575 from NIH. We acknowledge with thanks the cooperation of the staff of the Barrow Neurological Institute and Dr Donald Urrea of Mesa, Az.

\section{References}

Bender, M. (1952). Disorders in Perception. Thomas: Springfield, Ill.

Critchley, M. (1949). The phenomenon of tactile inattention with special reference to parietal lesions. Brain, 72, 538-561.

Critchley, M. (1953). The Parietal Lobes. Hafner: New York.

Eidelberg, E., and Schwartz, A. S. (1971). Experimental analysis of the extinction phenomenon in monkeys. Brain, 94, 91-108.

Gainotti, G., Caltagirone, C., Lemmo, M. A., and Micelli, G. (1975). Pattern of ipsilateral clinical extinc- 
tion in brain-damaged patients. Applied Neurophysiology, 38, 115-125.

Heilman, K. M., and Valenstein, E. (1972). Frontal lobe neglect in man. Neurology (Minneap.), 22, 660-664.

Krueger, E. G., Price, P. A., and Teuber, H. L. (1954). Tactile extinction in parietal lobe neoplasm. Journal of Psychology, 38, 191-202.

Nathan, P. W. (1946). On simultaneous bilateral stimulation of the body in a lesion of the parietal lobe. Brain, 69, 325-334.
Schwartz, A. S., and Eidelberg, E. (1968). 'Extinction' to bilateral simultaneous stimulation in the monkey. Neurology (Minneap.), 18, 61-68.

Teuber, H.-L. (1975). Effects of focal brain injury on human behavior. In The Nervous System: vol. 2; The Clinical Neurosciences, pp. 457-480. Edited by T. N. Chase. Raven Press: New York.

Welch, K., and Stuteville, P. (1958). Experimental production of unilateral neglect in monkeys. Brain, 81, 341-34i. 